\title{
Energy Optimisation in Wireless Sensor Network
}

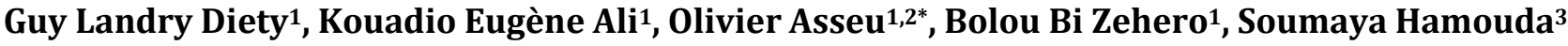 \\ ${ }^{1}$ Institute National Polytechnique Houphouët Boigny (INPHB), Yamoussoukro, Côte d'Ivoire \\ ${ }^{2}$ Ecole Supérieure Africaine des TIC (ESATIC), Abidjan, Côte d'Ivoire \\ ${ }^{3}$ Ecole Supérieure des Communications de Tunis (Sup'Com Tunis), Tunis, Tunisie \\ Email: *oasseu@yahoo.fr
}

How to cite this paper: Diety, G.L., Ali, K.E., Asseu, O., Bi Zehero, B. and Hamouda, S. (2017) Energy Optimisation in Wireless Sensor Network. Engineering, 9, 880889.

https://doi.org/10.4236/eng.2017.910053

Received: September 25, 2017

Accepted: October 24, 2017

Published: October 27, 2017

Copyright (c) 2017 by authors and Scientific Research Publishing Inc. This work is licensed under the Creative Commons Attribution International License (CC BY 4.0).

http://creativecommons.org/licenses/by/4.0/

\begin{abstract}
The solution we propose optimizes the energy inside the wireless sensor network (WSN) with higher performance. The WSN is composed of many sensors nodes which collect the information, treat that information then send it to the base station. The information is received by the base station (BS) then data are sent to the users by that BS. The most important element in sensor node is energy, as the lifetime of wireless sensor network depends on the sensor node energy. So many researches had been made in order to improve this energy basing routing protocols. As a result, we are able to propose a solution that optimizes this energy. In this paper, we are presenting a new approach of selecting node sensor base on routing protocol and process to send data to the base station. This ameliorates wireless sensor network lifetime and increases the transmission sensor node to base station.
\end{abstract}

\section{Keywords}

Wireless Sensor Network (WSN), Base Station (BS), Sensor Node (SN), Energy, Routing Protocol, Transmission, Optimisation

\section{Introduction}

The apparition of internet of things did change many things in the field of technologies. The Internet of Things (IoT) technology permits to connect anything like house, office, hospital, road, car etc. and man to internet. We can find internet of things in many domains which are for example army, medicine, automobile, house, industries and others. It participates in creating smart environments.

The sensor network, particularly the WSN is a very important thing in this technology. Indeed, WSN is composed of sensors nodes which can receive data, treat the data and send it to the base station (BS), then BS at its turn, sends the 
information to the users.

Basically, each sensor node comprises sensing, processing, transmission, mobilizer, position finding system, and power units (some of these components are optional like the mobilizer). Some popular cluster based routing schemes towards achieving the required goal of energy efficiency to prolong network lifetime are discussed here in brief: The LEACH [1], introduced by Heizelman et al., is one of the most popular clustering based routing protocols and most of the other clustering schemes proposed in the literature are aimed at enhancing energy efficiency at one or more phases in LEACH. LEACH determines a fraction $\mathrm{p}$ of all the sensor nodes that may become cluster heads a priori.

Energy efficient announcement is very important to extend the sensor networks. In recent times, different routing protocols have been reported concerning wireless sensor networks. Many protocols apply single optimal route in support of data transmission.

The optimal route is selected based on the metrics, such as minimum hop, high remaining energy, minimum broadcast etc. to route the data [2] [3]. Routing is very challenging in wireless sensor networks. One of the most difficulties that involve the sensor network lifetime refers to sensor nodes in the neighbourhood of the sensor sink, whose activity affects a high traffic on this sequence of sensor nodes [4].

Nodes in networks are grouped into clusters, and nodes that have higher remaining energy are selected as the cluster heads $(\mathrm{CH})$. In each cluster, the nominated $\mathrm{CH}$ node receives and aggregates data from all the sensor nodes in the cluster. Usually, the sizes of the data off all the sensor nodes are the same, and the aggregated in data at the $\mathrm{CH}$ node has the same size with the data of every sensor in the cluster. As the data are aggregated in the $\mathrm{CH}$ node before reaching a BS (base station), this technique reduces the amount of information sent to the distant BS, hence saves energy. For example, if each sensor in the cluster sends a message of 100 bits to the $\mathrm{CH}$ node, then the $\mathrm{CH}$ node sends the aggregated message of 100 bits to the BS [8]. Details are given in [1] [5] [6].

When in LEACH cluster-head are selected randomly, EECS selects a node as a cluster-head with the highest residual energy among all the nodes, competing in order to be a cluster head. Many types of existing routing protocols are developed to save power consumption. In these protocols, cluster based routing protocols are found to be more energy efficient. A cluster head is selected to aggregate the data received from rout nodes and forward that data to the base station in cluster based routing [7].

By virtue of heterogeneity in terms of energy, the network lifetime can be extended. An algorithm is functional if the area of interest is covered by active nodes. The period network functioning period is termed as persistent period [8].

The connection between destination and task manager node is the public networks in the form of satellite. Data are received from the task manager node by the ending users who perform processing on received data [9]. Energy plays 
effective role in wireless sensor network. There are various challenges for deploying wireless sensor network like scalability, programmability maintainability, quality of services, types of service and lifetime of the network [10].

According to the thing we want to follow, several sensors nodes can be deployed in perimeter or zone to cooperate on sensing a physical phenomenon. Sensor networks hold the promise of revolutionizing sensing in a wide range of application domains because of their reliability, accuracy, flexibility, cost-effectiveness, and ease of deployment [11]. At network layer, several approaches have already been followed by the researchers towards developing energy efficient routing schemes like viz. flat routing, hierarchical routing, location based routing etc. Among these approaches hierarchical routing sounds to be the most attractive as many algorithms have been developed treating this as a base. It may not always provide any assurance either about the number of cluster head nodes or how well these clusters are [11].

Ravi Tomar did criticize and made an important improvement to EEPSC from LEACH. According to him, although his critics as well as the improvement are valid, we still affirm it is not really sufficient. The difference in terms of lifetime between WSN from EEPSC and WSN from Ravi Tomar is very few; it is not sufficient and efficient in transmitting more data to the base station. On the contrary, our approach presented in this paper does obviously help making WSN lifetime longer in order to better transmit data to the base station.

Indeed, our proposed method increases data sent to the base station and make lifetime of WSN better. We select sub-cluster heads among the cluster heads. This avoids the quickly death of the Wireless Sensor Nodes. Its activities are reduced by delegating transmission to its sub-clusters head according to the event. This improves lifetime of WSN and permits to send more data to the base station.

\section{Method and Process}

Delia Ciullo, Guner D. Celik and Eytan Modiano introduce and analyse the problem of optimal vehicle trajectories as a function of the amount of data at each sensor in order to minimize the total transmission energy within a given travel time limit [12]. As for Shubhi Bansal and Vishal Kumar, they propose a combination of centralized and distributed clustering approach. For carrying out efficient clustering, they have divided the network area into two zones (nearer zone and farther zone). Both the distributed and centralized clustering have their advantages and disadvantages [13]. While Mahmuda Nazin and Sayed Chowdhury propose an energy efficient data gathering method. The tasks of data collection are distributed among the nodes of the different zones which resist the sensor nodes from going to the bottleneck state by minimizing latency [14]. A. B. M. Al Islam, Chowdhury Sayeed Hyder, Humayun Kabir and Mahmuda Naznin for their part propose a new and complete mathematical model on LEACH. They calculate the value for the optimical percentage of cluster heads in order to increase the network lifetime [15]. Nizar Hadi, Tarik Zeyad and Rassim 
Nooraldin propose a method based on mathematical model for lifetime of WSN with several parameters to find out optimal solution of the energy problem in the field of WSN using the modified particle swarm optimization and ant colony optimization algorithms [16]. And then Banakar Vinodkumar and Mrs. Geetha N B and Mohamed Rafi consider the cost of sending as well as processing. So, they use short distance path as well as compression of the data to reduce the power consumption. They design and implement TARF, a robust trust-aware routing framework for dynamic WSNs. Without tight time synchronization or know geographic information, TARF provides trustworthy and energy-efficient route [17].

Our approach is very different from all methods mentioned before. Our approach is to improve the protocol LEACH model Ravi Tomar which inspires protocol EEPSC (Energy-Efficient protocol with static clustering).

The selection of the cluster head is made by compared residual energy of all sensor nodes in the sub-area. The sensor node whose energy is higher than the others' is designed as cluster head. With Ravi Tomar's protocol, there is a new function added. We must firstly compare residual energy and secondly centralize the sensor node according to all the sensor nodes in the area. After a round, another cluster head is selected.

Ravi Tomar's protocol is an improvement of the protocol EEPSC [1]. Ravi Tamar's protocol prefers to centralize the cluster head selected. But the inconvenient is the cluster head will be more solicited and will quickly die, because it will be the only one which works every time.

To avoid the node quickly death inside the WSN, we propose in Figure 1, to increase lifetime by selecting some sub-cluster head around the cluster heads selected. Doing this, we reduce more the activities of the cluster head by delegation of transmission to sub-cluster head to base station. We add a new function which is the distance between the event, cluster head, sub-cluster head and base

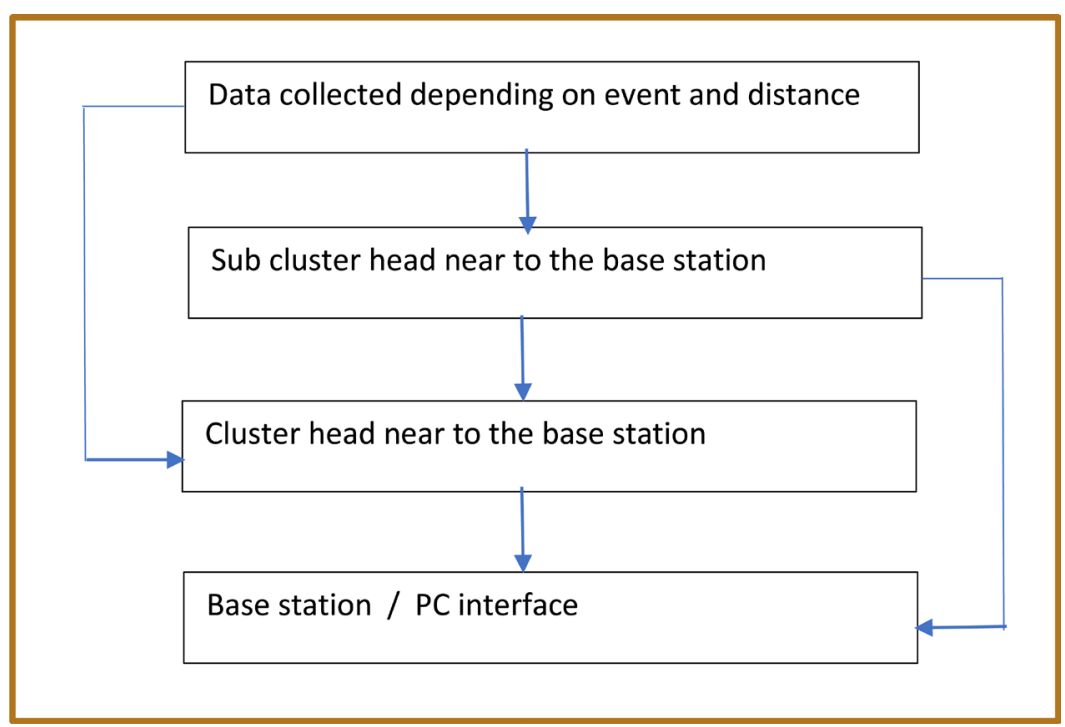

Figure 1. Data sending process. 
station. According to the event, if the distance between cluster head-base station is more than distance sub-cluster head-base station, then the sub-cluster head transmit to a neighbour and join the base station later.

This ameliorates the WSN lifetime, all sub-cluster heads work together in synchronized time to help cluster head at a periodical time with less energy. We have more transmissions and less dead nodes. Our sending process is described by the follow scheme.

\section{Process to Select Cluster Head and Sub-Cluster Head}

Our task consists in improving Ravi Tomar's protocol. So, we should work with he's same conditions (parameters) and prove that we did a good improve WSN's lifetime.

a) Cluster Head selection

Let $n, p, r \in N^{*}$

Let $A=\{1,2, \cdots, q\}$ the set of nodes of zone $\Omega$ rectangular of length $X_{m}$ and width $Y_{m}$ (not Cluster Head).

$P_{\Omega}=\left\{\Omega_{i}, i \in\{1,2, \cdots, q\} / \Omega_{1} \cup \Omega_{2} \cup \cdots \cup \Omega_{q}=\Omega\right.$ and $\Omega_{i} \cap \Omega_{j}=\varnothing$ if $\left.i \neq j\right\} \quad$ a partition of $\Omega$.

We denote $A_{i}$ the set of nodes of zone $\Omega_{i}$

$P_{A}=\left\{A_{i}, i \in[1, q]\right.$, like $A_{1} \cup A_{2} \cup \cdots \cup A_{q}=A$ with $A_{i} \in \Omega_{i}$ and $A_{i} \cap A_{j}=\varnothing$ if $\left.i \neq j\right\}$

We denote $C H_{i}$ the cluster head of zone $\Omega_{i}$ and $S C H_{i}$ a sub-cluster head of the zone $\Omega_{i}$.

Each node $K$ of $\Omega$ generate a random number $r n(k)$ comprise on 0 and 1 .

The eligibility threshold value of $\mathrm{CH}_{i}$ is defined by:

$$
T(k)=\frac{P}{1-P * r \bmod \left(\frac{1}{P}\right)} \text { if } k \in A \text { and } T(k)=0 \text { if } k \notin A
$$

where $P$ is the percentage of cluster heads zone, $r$ is the number of rounds.

If $r n(k) \geq T(k)$ then the node $k$ is eligible.

For any $\mathrm{CH}_{i}$ and $\mathrm{CH}_{j}$ distinct it's necessary that:

$$
d\left[\mathrm{CH}_{i}, \mathrm{CH}_{j}\right] \geq \sqrt{\frac{X_{m} * Y_{m}}{K o p t}}=M S D
$$

d: distance; $M S D$ ((minimal distance between two clusters head).

where $X_{m}, Y_{m}$ are the length and width of $\Omega$ respectively, Kopt is the optimal number of cluster head, $d(x, y)$ is the distance between $x$ and $y$.

b) Sub-Cluster Head selection

The eligibility threshold value of $S C H_{i}$ is defined by:

$$
T_{s}(k)=\frac{P_{s}}{1-P_{s} * r \bmod \left(\frac{1}{P_{s}}\right)} \text { if } k \in A \text { and } T_{s}(k)=0 \text { if } k \notin A
$$

where $P_{s}$ is the percentage of sub-cluster heads zone, $r$ is the number of rounds. 
If $r n(k) \geq T_{s}(k)$ then the node $k$ is eligible to be sub-cluster head.

Let $S_{i}$ be the set of nodes in $A_{i}-\left\{C H_{i}\right\}$ which verify relation (3).

Then the $m_{i}$ elements of $S_{i}$ which energies are better are the $S C H_{i}$.

If $S_{i}=\phi$ then the $S C H_{i}$ are the elements of $A_{i}-\left\{C_{i}\right\}$ verifying energy condition. Even if $S_{i}$ is empty, we select a few nodes which random value and energy value are the closest to the threshold value.

c) Transmission method

We define the following distance to the Base Station (BS):

$$
d\left(C H_{i}, B S\right)=\sqrt{\left(X_{C H i}+X_{B S}\right)^{2}+\left(Y_{C H i}+Y_{B S}\right)^{2}}
$$

$d\left(\mathrm{CH}_{i}, \mathrm{BS}\right)$ the distance from $\mathrm{CH}_{i}$ to the $B S$.

$$
d\left(\mathrm{SCH}_{i}, \mathrm{BS}\right)=\sqrt{\left(X_{\mathrm{SCHi}}+X_{B S}\right)^{2}+\left(Y_{S C H i}+Y_{B S}\right)^{2}}
$$

$d\left(S_{C H}, B S\right)$ the distance from $S C H_{i}$ to the $B S$

Let $\Omega_{e}$ be the zone where the event happened. If $\Omega_{e}$ is far from the base station, we choose $\Omega_{\mathrm{j}}$ zone in the neighbourhood zone of $\Omega_{e}$ which contains the cluster head or the sub-cluster head closer to the base station.

The information must firstly be transmitted from $\Omega_{e}$ to $\Omega_{j}$. Thus, the cluster head or sub-cluster head nearest to the event transmits the information to the cluster head or sub-cluster of $\Omega_{e}$ nearest to the cluster head or sub-cluster head $\Omega_{j}$ that is nearest to the base station. The process goes on up to the zone nearest to the base station. Within that last zone, the cluster head or the sub-cluster head nearest to the base station transmits the information to the base station.

If $\Omega_{e}$ is the zone nearest to the base station, then the information is transmitted by the cluster head or the sub-cluster head of $\Omega_{e}$ nearest to the event to the cluster head or the sub-cluster head nearest to the base station. The information is finally sent to the base station by the cluster head or sub-cluster head nearest to that base station.

In Figure 2, we propose a method that permits the cluster head to have a longer lifetime and much more avoid the death of sensor node in the network. With this method, we get a better result than the result from protocol LEACH, protocol EEPSC and Ravi Tomar's protocol.

\section{Simulation and Results}

To compare two methods, we must do some experiences with them in the same conditions considering the software used, environment, number of sensor nodes. Table 1 resumes all the parameters used. The simulation gives many results about lifetime of WSN, number of packets transmitted to base station and cluster heads.

\section{a) Network lifetime per round}

The simulation gives many results about the WSN lifetime, number of packets transmitted to base station and cluster heads.

We observe the stability of the network (the interval time from the start of 


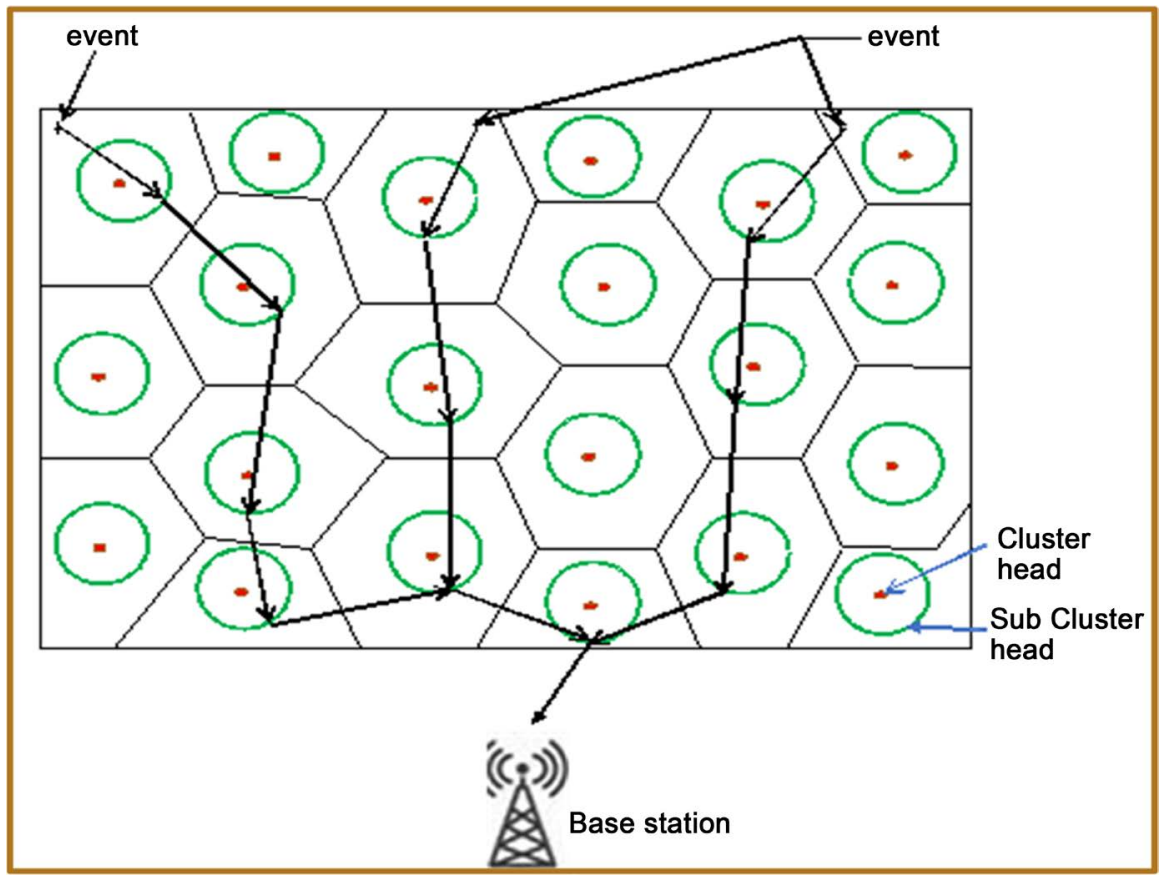

Figure 2. Transmission process from cluster head and sub-cluster to the base station.

Table 1. Parameter settings.

\begin{tabular}{ccc}
\hline Parameter & Meaning & Values \\
\hline$M \times N$ & Network Area Dimensions & 100 meter $\times 100$ meter \\
$N$ & Number of SNs & 100 \\
$E_{\text {elec }}$ & Electronic energy & $50 \mathrm{~nJ} / \mathrm{bit}$ \\
$E D A$ & Data aggregation energy & $5 \mathrm{~nJ} / \mathrm{bit} / \mathrm{message}$ \\
$E_{0}$ & Initial energy & $0.5 \mathrm{~J}$ \\
$k$ & Size of the message & $4000 \mathrm{bits}$ \\
$P_{\text {opt }}$ & Probability & 0.05 \\
$\varepsilon_{f s}$ & Friss free space & $10 \mathrm{pJ} / \mathrm{bit} / \mathrm{m}^{2}$ \\
$\varepsilon_{m p}$ & Friss multi-path & $0.0013 \mathrm{pJ} / \mathrm{bit} / \mathrm{m}^{4}$ \\
$a$ & Energy level for Advance nodes & 3 \\
$b$ & Energy level for Intermediate nodes & 1.5 \\
$m, X$ & of nodes to be Advance and Intermediate & $0.2,0.3$ \\
$B S$ & Base Station Location & $(500,500)$ \\
\hline
\end{tabular}

network operation and the death of sensor node) after 10,000 rounds in Figure 3. All sensor nodes from LEACH and from Ravi Tomar's protocol die quickly after 2500 rounds. The stability period and network lifetime of LEACH is less as compared to Ravi Tomar's and DIETY's. Our protocol called DIETY keeps sensor nodes alive to 9500 rounds. And then we can transmit more data to base station. The protocol DIETY is really better because of its awareness in terms of energy heterogeneity and the use multi-hoping concept in it. 


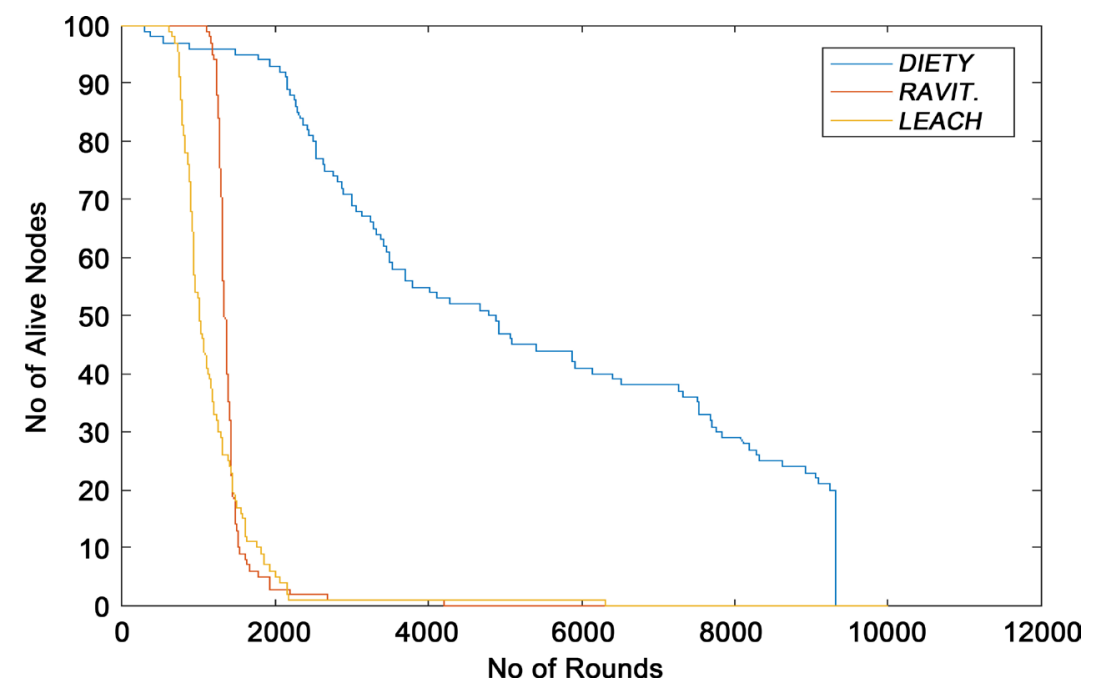

Figure 3. Dead nodes comparison DIETY, RAVI T. and LEACH.

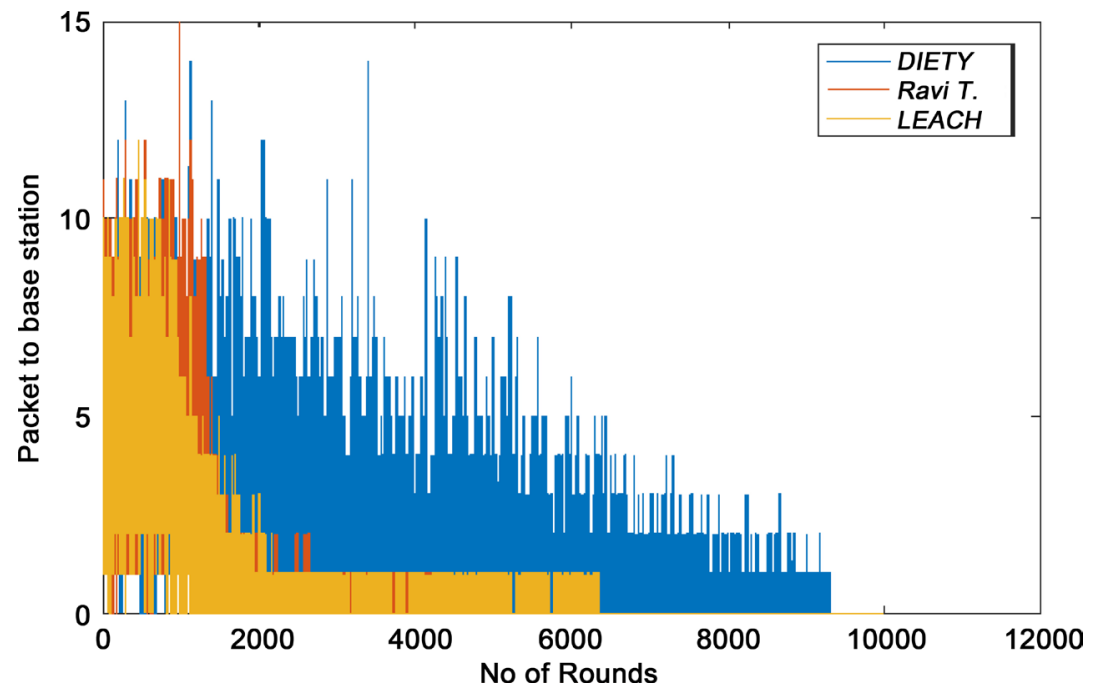

Figure 4. Packets transmitted to the base station.

\section{b) Number of packets transmitted to base station}

Depending on the lifetime of WSN, with our method, we transmit more data to the base station. The Figure 4 and Figure 5 show the number of packets transmitted to BS per round. It is found that DIETY provides continuous $\mathrm{CH}$ and have the highest successful data rate as compared to RAVI T. and LEACH for more number of rounds. LEACH and Ravi T. performed poorly and failed to provide optimal number of $\mathrm{CHs}$ due to their distributed nature of selecting $\mathrm{CHs}$. After 2.500 rounds there is no possibility to send packet to BS because of dead sensor node with LEACH and Ravi Tomar's. DIETY continuous to send packet to BS because of the repartition method between cluster head and sub-clusters heads. Also, to analyse the quality of any routing protocol, the successful data transmission of packets to BS is a very important parameter. So, if BS is receiving high amount of data then that routing protocol is working properly.

Due to this uneven generation of CHs per rounds for $\mathrm{LEACH}$ and Ravi T. 


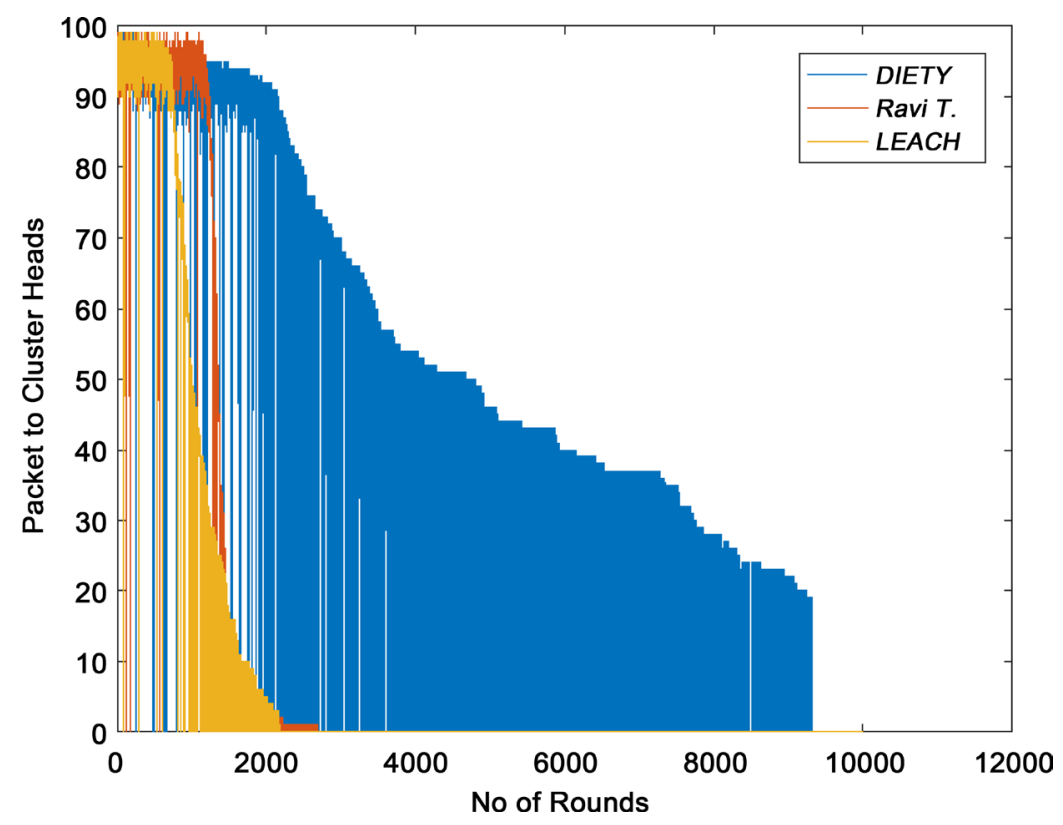

Figure 5. Packets to cluster head and sub-cluster heads.

shows the negative impact on the handling of network traffic which then results in the loss of packets due to insufficient TDMA slots. Also, DIETY is stable when number of $\mathrm{CH}$ varies from $30-50$, because if we increase the number of $\mathrm{CH}$ (included sub-cluster head becomes cluster head to transmits) then it results in that non- $\mathrm{CH}$ nodes can easily get $\mathrm{CH}$ locally and perform data aggregation.

\section{Conclusions}

Our method and approach show that we can get better result than Ravi Tomar's method. According to this approach, all sensor nodes corporate and we have more cluster head (included sub-cluster head) to send more packets to the base station.

The WSN lifetime becomes longer and dead sensor nodes are more reduce. Our method is not the only best one, but it contributes to improve or increase then lifetime of WSN. Having more lifetime of WSN proves that we can get more information about internet of things because sometimes data has been sending by WSN.

\section{References}

[1] Heinzelman, W.R., Chandrakasan, A. and Balkrishnan, H. (2000) Energy-Efficient Communication Protocol for Wireless Microsensor Networks. Proceeding of 33 rd Hawaii International Conference on System Science, 2, 10 https://doi.org/10.1109/HICSS.2000.926982

[2] Erdene-Ochir, O., et al. (2010) Resilient Routing in Wireless Sensor Networks: Gradient-Based Routing in Focus. IEEE of Fourth International Conference on Sensor Technologies and Applications (SENSORCOMM), July 2010, 478-483. https://doi.org/10.1109/SENSORCOMM.2010.77

[3] Cheng, L., Das, S.K, Cao, J.N., Chen, C.F. and Ma, J. (2010) Distributed Minimum 
Transmission Multicast Routing Protocol for Wireless Sensor Networks. IEEE 39th International Conference on Parallel Processing (ICPP), 188-197.

[4] Arya, R. and Sharma, S.C. (2015) Analysis and Optimization of Energy of Sensor Node Using ACO in Wireless Sensor Networks. International Conference on Advanced Computing Technologies and Applications (ICACTA), 681-686.

[5] Nguyen, T.T. and Nguyen, V.D. (2012) Optimizing the Operating Time of Wireless Sensor Network. EURASIP Journal on Wireless Communication and Networking. https://doi.org/10.1186/1687-1499-2012-348

[6] Nguyen Thanh, T. (2012) Heuristic Energy-Efficient Routing Solutions to Extend the Lifetime of Wireless Ad-Hoc Sensor Networks. Springer, 487-497.

[7] Khan, Z.A. and Sampalli, S. (2012) AZR-LEACH: An Energy Efficient Routing Protocol for Wireless Sensor Networks. International Journal Communications, Networks and System Sciences, 785-795.

[8] Mehra, P.S., Doja, M.N. and Alam, B. (2015) Energy Efficient Self Organising Load Balanced Clustering Scheme for Heterogeneous WSN. 2015 International Conference on IEEE Energy Economics and Environment (ICEEE).

[9] El Khediri, S. and Nasri, N. (2014) A New Approach for Clustering in Wireless Sensor Networks Based on LEACH.

[10] Manisha and Mittal, N. (2016) Deterministic Relay Node Based Improved LEACH Protocol for Wireless Network: A Review. International Journal for Innovative Research in Science \& Technology, 3.

[11] Tomar, R. (2014) Qos Issues in Wireless Sensor Networks: An Energy Efficient Approach. LAP LAMBERT Academic Publishing, Saarbrücken.

[12] Ciullo, D., Celik, G.D. and Modiano, E. (2010) Minimizing Transmission Energy in Sensor Networks via Trajectory Control.

http://www.mit.edu/ modiano/papers/C141.pdf

[13] Bansal, S. and Arora, V.K. (2015) Energy Enhancement Using Area Zoning in Wireless Sensor Networks. $2^{\text {nd }}$ International Conference on Science, Technology and Management, New Delhi, 27 September 2015.

[14] Naznin, M. and Chowdhury, A.S. (2015) Energy Efficient Zone Based Data Gathering in a Wireless Sensor Network.

[15] Alim Al Islam, A.B.M., Hyder, C.S., Kabir, H. and Naznin, M. (2010) Finding the Optimal Percentage of Cluster Heads from a New and Complete Mathematical Model on LEACH. Wireless Sensor Network, 2, 129-140. https://doi.org/10.4236/wsn.2010.22018

[16] Abbas, N.H., Ismaeel, T.Z. and Ibrahim, R.N. (2013) Optimization of Energy Consumption in Wireless Sensor Networks based on Nature-Inspired Algorithms. International Journal of Computer Applications, 77, 32-39. https://doi.org/10.5120/13553-1345

[17] Vinodkumar, B., Geetha, N.B. and Rafi, M. (2015) Energy Optimization in Wireless Sensor Networks. International Journal of Computer Science and Mobile Computing, 4, 549-553. 